\title{
Number of Frames
}

National Cancer Institute

\section{Source}

National Cancer Institute. Number of Frames. NCI Thesaurus. Code C69267.

The total number of frames in a multiframe image. 